\title{
Morphological Complexity of Protostellar Envelopes
}

\author{
John J. Tobin ${ }^{1}$, Lee Hartmann ${ }^{1}$, Edwin Bergin ${ }^{1}$, Leslie W. Looney ${ }^{2}$, \\ Hsin-Fang Chiang ${ }^{2}$, Fabian Heitsch ${ }^{3}$ \\ ${ }^{1}$ Department of Astronomy, University of Michigan, Ann Arbor, MI 48109; jjtobin@umich.edu \\ ${ }^{2}$ Department of Astronomy, University of Illinois at Champaign/Urbana, Urbana, IL 61801 \\ ${ }^{3}$ Department of Physics and Astronomy, University of North Carolina-Chapel Hill, Chapel \\ Hill, NC
}

\begin{abstract}
Extinction maps at $8 \mu \mathrm{m}$ from the Spitzer Space Telescope show that many Class 0 protostars exhibit complex, irregular, and non-axisymmetric structure within the densest regions of their dusty envelopes. Many of the systems have highly irregular and non-axisymmetric morphologies on scales $\sim 1000 \mathrm{AU}$, with a quarter of the sample exhibiting filamentary or flattened dense structures. Complex envelope structure is observed in regions spatially distinct from outflow cavities, and the densest structures often show no systematic alignment perpendicular to the cavities. We suggest that the observed envelope complexity is the result of collapse from protostellar cores with initially non-equilibrium structures. The striking non-axisymmetry in many envelopes could provide favorable conditions for the formation of binary systems. We then show that the kinematics around L1165 as probed with $\mathrm{N}_{2} \mathrm{H}^{+}$are indicative of asymmetric infall; the velocity gradient is not perpendicular to the outflow.
\end{abstract}

Keywords. stars: formation, dust, extinction, ISM: molecules, ISM: globules

\section{Introduction}

Sphericity and axisymmetry have been standard assumptions on which our theoretical understanding of star formation has rested for some time (e.g. Shu 1977; Terebey et al. 1984; Galli \& Shu 1993; Hartmann et al. 1996). However, it is not clear whether or not envelopes around protostars are accurately described by symmetric models. The shapes of dense cores have been studied on large scales $(>0.1 \mathrm{pc})$ using molecular line tracers of dense gas (Benson \& Myers 1989; Myers et al. 1991). While the ammonia cores appeared compact and round due to low resolution, associated IRAS sources are often located offcenter from the line emission peaks indicating non-axisymmetry. Furthermore, emission from the millimeter-line tracers appears quite complex on scales outside the ammonia emission(Myers et al. 1991).

Recently, observations with the Spitzer Space Telescope have given a high-resolution view of envelope structure in extinction at $8 \mu \mathrm{m}$ against Galactic background emission (Looney et al. 2007; Tobin et al. 2010). This method enables us to observe the structure of collapsing protostellar envelopes on scales from $\sim 1000 \mathrm{AU}$ to $0.1 \mathrm{pc}$ for the first time with a mass-weighted tracer. In contrast, single-dish studies of envelopes using dust emission in the sub/millimeter regime generally have lower spatial resolution. The continuum emission depends upon temperature and density, while molecular tracers are additionally affected by complex chemistry.

In this contribution, we present IRAC $8 \mu \mathrm{m}$ extinction maps of envelopes around Class 0 protostars. Most of the envelopes in our sample are found to be irregular and nonaxisymmetric. We suggest that these envelopes may be important for binary formation 

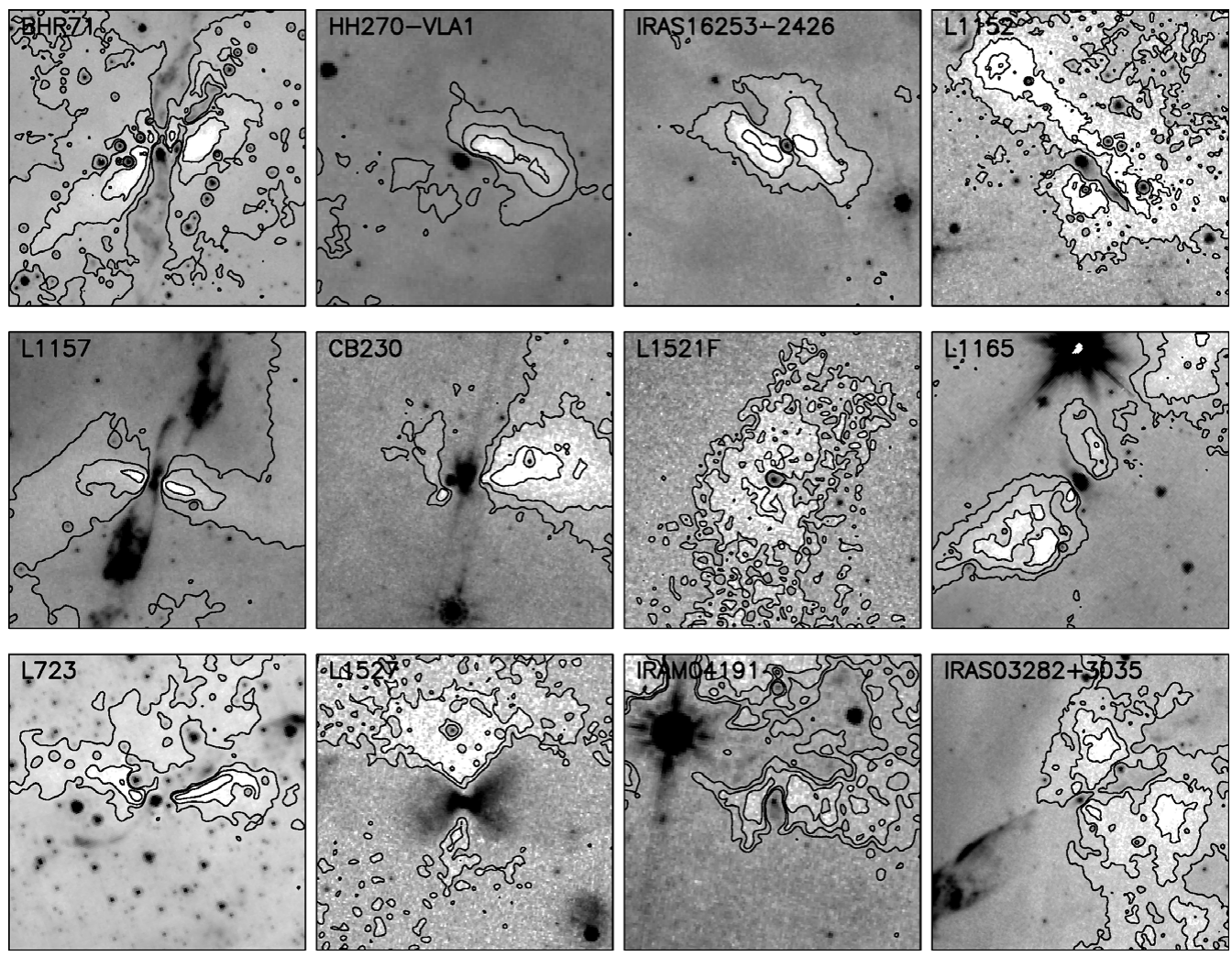

Figure 1. IRAC $8 \mu \mathrm{m}$ images with $8 \mu \mathrm{m}$ optical depth contours overlaid for a selection of the protostars from Tobin et al. (2010). From left to right the columns are filamentary envelopes, one-sided envelopes, quasi-symmetric envelopes, and irregular envelopes respectively.

as collapse models have shown that asymmetric structure can induce small-scale fragmentation of the infalling envelope. We also show initial results in analyzing the kinematic structure of these envelopes using the dense gas tracers $\mathrm{N}_{2} \mathrm{H}^{+}$.

\section{Asymmetric Envelopes in Extinction}

In Figure 1, we display a selection of 12 systems (out of 22 from Tobin et al. (2010)) for which we have detected an envelope in extinction. Each panel shows the $8 \mu \mathrm{m}$ image of the protostar with optical depth contours overlaid. In most images, the outermost extinction contour corresponds to $\mathrm{A}_{V} \sim 5-10$ and going up to $\mathrm{A}_{V} \sim 30$.

The most striking feature of the $8 \mu \mathrm{m}$ extinction maps is the irregularity of envelopes in the sample. Most envelopes show high degrees of non-axisymmetry; in most cases, spheroids would be an inaccurate representation of the structure. Some of the most extreme examples have most extincting material mostly on one side of the protostar (e.g. CB230, HH270 VLA1) or the densest structures are curved near the protostar (e.g. BHR71, L723). The structures seen in extinction at $8 \mu \mathrm{m}$ do not seem to be greatly influenced by the outflow. Tobin et al. (2010) demonstrated that the outflow cavities of these sources are generally quite narrow and the dense material detected in extinction is often far from the outflow cavities and thus unlikely to be produced by outflow effects.

For convenience, we categorize the systems into 4 groups according to their morphology, though some systems have characteristics of multiple groups. The first column of Figure 1 shows the envelopes that have a highly filamentary/flattened morphologies; column 2 

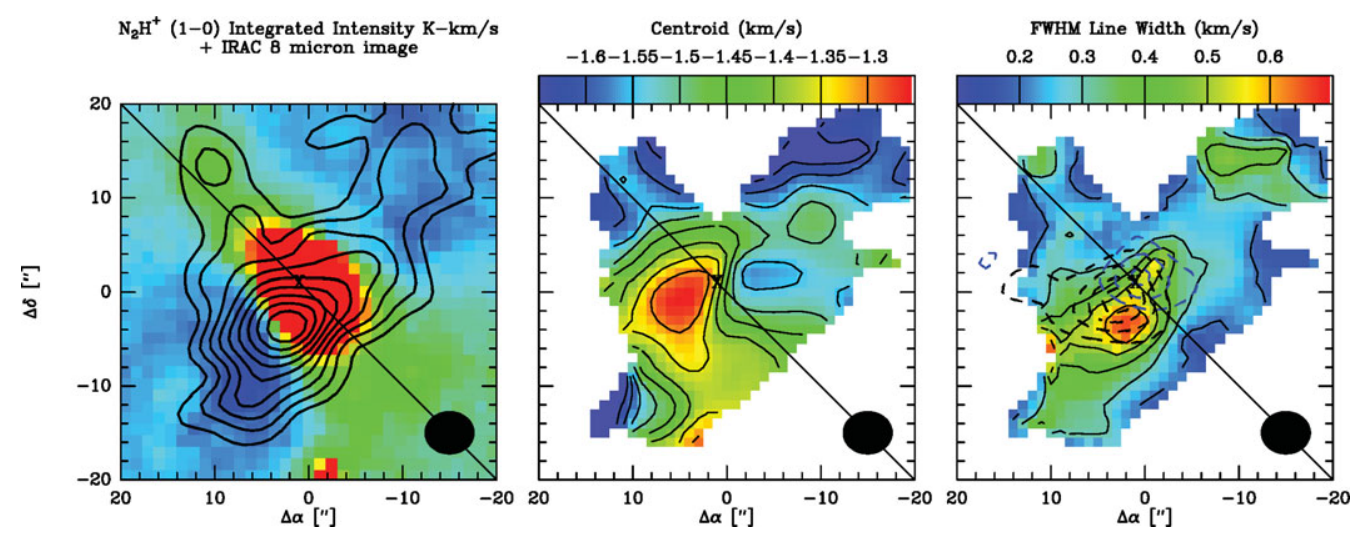

Figure 2. $\mathrm{N}_{2} \mathrm{H}^{+}(J=1 \rightarrow 0)$ observations for the protostar L1165; in all panels the protostar is marked with an $\mathrm{X}$ and the outflow axis is marked with a straight line. Left: IRAC $8 \mu \mathrm{m}$ image (color scale) with $\mathrm{N}_{2} \mathrm{H}^{+}(J=1 \rightarrow 0)$ integrated intensity (contours). Middle: Centroid velocity of $\mathrm{N}_{2} \mathrm{H}^{+}$emission derived from fitting the hyperfine lines; notice the sharp gradient across the protostar which is not perpendicular to the outflow. Right: Full-width half maximum of $\mathrm{N}_{2} \mathrm{H}^{+}$ lines; note that the linewidth is greatest at the peak of $\mathrm{N}_{2} \mathrm{H}^{+}$emission. The dashed contours in the rightmost panel are the $\mathrm{HCO}^{+}$blue (blue) and redshifted (black) emission.

shows envelopes that have most material on one side of the protostar; column 3 shows the protostars whose envelopes are more or less spheroidal in projection; column 4 shows envelopes that do not strictly fall within the above categories.

\section{Kinematic Structure}

In addition to morphological structure, the kinematic structure of the envelope resulting from the non-axisymmetric envelope is of great importance for the subsequent evolution of the system. Figure 2 shows the $\mathrm{N}_{2} \mathrm{H}^{+}(J=1 \rightarrow 0)$ observations for the protostar L1165 from the CARMA millimeter array. The $\mathrm{N}_{2} \mathrm{H}^{+}$emission shows that the envelope is elongated along the larger filament consistent with the IRAC $8 \mu \mathrm{m}$ image and the peak emission is offset from the protostar; the envelope also remains filamentary down to small scales. The centroid velocity map shows a strong velocity gradient across the envelope, $0.35 \mathrm{~km} / \mathrm{s}$ over $2400 \mathrm{AU}$; though, the linewidth indicates that the gradient could be larger. At present, it is difficult to differentiate between projected infall or rotation giving rise to the velocity structure; in either case the infall motions will be complex. Given that the $\mathrm{N}_{2} \mathrm{H}^{+}$velocity gradient is $\sim 30^{\circ}$ from perpendicular to the outflow and the $\mathrm{HCO}^{+}$gradient is perpendicular it seems possible that the angular momentum vector may change with time.

\section{Implications of Non-Axisymmetric Collapse}

The envelope asymmetries may result from the initial cloud structure. Stutz et al. (2009) recently surveyed pre-stellar/star-less cores using 8 and $24 \mu \mathrm{m}$ extinction; their results, and those of Bacmann et al. (2000), showed that even pre-collapse cloud cores already exhibit asymmetry. Given the initial asymmetries, the densest, small-scale regions are likely to become more anisotropic during gravitational collapse (Lin et al. 1965).

\subsection{Binary Formation}

The smallest scales we observe, $\sim 1000 \mathrm{AU}$, is where angular momentum will begin to be important as the material falls further in onto the disk; this is the scale we probe 
with the $\mathrm{N}_{2} \mathrm{H}^{+}$observations of L1165. The envelope asymmetries down to small scales imply that infall to the disk will be uneven; therefore, non-axisymmetric infall may play a significant role in disk evolution and the formation of binary systems. Several theoretical investigations (e.g. Burkert \& Bodenheimer 1993) showed that collapse of a cloud with just a small azimuthal perturbation can form binary or multiple systems; thus, large non-axisymmetric perturbations should make fragmentation even easier. Fragmentation can even begin before global collapse in a filamentary structure (Bonnell et al. 1993). Numerical simulations of disks with infalling envelopes (e.g. Kratter et al. 2009) informed by the results of this study could reveal a more complete understanding of how nonaxisymmetric infall affects the disk and infall process. Asymmetric infall may be taking place in at least L1165. However, it is not known if this system is a binary because the requisite high-resolution observations have not been taken.

\subsection{Initial Conditions}

The envelopes we have shown are not obviously consistent with quasi-static, slow evolution, which might be expected to produce simpler structures as irregularities have time to become damped; one needs initial non-axisymmetric structure to get strong nonaxisymmetric structure later on. This raises the question of the role of magnetic fields in controlling cloud dynamics. In some models (e.g. Tassis et al. 2007), pre-stellar cores would probably live long enough to adjust to more regular configurations; in addition, collapse would be preferentially along the magnetic field, which would also provide the preferential direction of the rotation axis and therefore for the (presumably magnetocentrifugally accelerated) jets (Basu \& Mouschovias 1994). The complex structure and frequent misalignment between collapsed structures and outflows pose challenges for such a picture. Alternatively, this could indicate that the topology of magnetic field is complex and not initially well ordered.

In contrast, more recent numerical simulations (e.g. see review by Ballesteros-Paredes et al. 2007; Offner \& Krumholz 2009) suggest that cores are the result of turbulent fluctuations which naturally produce more complex structure with less control by magnetic fields amplified by subsequent gravitational contraction and collapse. Thus, the structure of protostellar envelopes indicates that the dynamic, turbulent model of rapid star formation seems more correct. However, recent work has indicated that turbulence can reduce the time needed for ambipolar diffusion to take place which may allow complex structures to form (Basu \& Ciolek 2009).

\section{References}

Ballesteros-Paredes, J., et al. 2007, Protostars and Planets V, 63

Bacmann, A., et al. 2000, A\&A, 361, 555

Basu, S., Ciolek, G. E., Dapp, W. B. \& Wurster, J. 2009, New Astronomy, 14, 483

Bonnell, I. \& Bastien, P. 1993, ApJ, 406, 614

Burkert, A. \& Bodenheimer, P. 1993, MNRAS, 264, 798

Galli, D. \& Shu, F. H. 1993, ApJ, 417, 220

Kratter, K. M., Matzner, C. D., Krumholz, M. R., \& Klein, R. I. 2010, ApJ, 708, 1585

Lin, C. C., Mestel, L., \& Shu, F. H. 1965, ApJ, 142, 1431

Looney, L. W., Tobin, J. J., \& Kwon, W. 2007, ApJL, 670, L131

Offner, S. S. R., \& Krumholz, M. R. 2009, ApJ, 693, 914

Shu, F. H. 1977, ApJ, 214, 488

Stutz, A. M., et al. 2009, ApJ, 707, 137

Terebey, S., Shu, F. H., \& Cassen, P. 1984, ApJ, 286, 529

Tobin, J. J., Hartmann, L., Looney, L. W., \& Chiang, H.-F. 2010, ApJ, 712, 1010 